PROFESIONALES Y HERRAMIENTAS PARA EL DESARROLLO LOCAL Y SUS SINERGIAS TERRITORIALES. EVALUACIÓN Y PROPUESTAS DE FUTURO IX Coloquio Nacional de Desarrollo Local del GTDL-AGE 

ANTONIO MARTÍNEZ PUCHE, XAVIER AMAT MONTESINOS, ISABEL SANCHO CARBONELL y DANIEL SANCHIZ CASTAÑO (EDS.)

\section{PROFESIONALES Y HERRAMIENTAS PARA EL DESARROLLO LOCAL Y SUS SINERGIAS TERRITORIALES. EVALUACIÓN Y PROPUESTAS DE FUTURO}

IX Coloquio Nacional de Desarrollo Local del GTDL-AGE

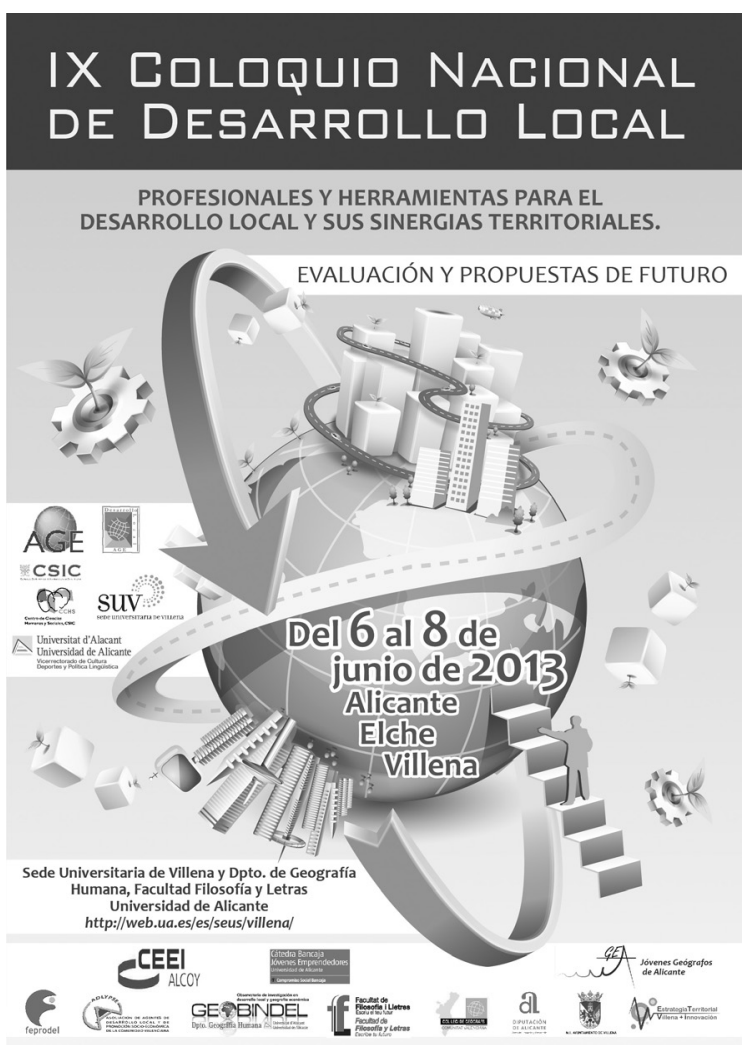


Este libro ha sido examinado y valorado por evaluadores ajenos a la Universidad de Alicante, con el fin de garantizar la calidad científica del mismo.

Publicacions de la Universitat d'Alacant

03690 Sant Vicent del Raspeig

Publicaciones@ua.es

http://publicaciones.ua.es

Telèfon: 965903480

(C) Antonio Martínez Puche, Xavier Amat Montesinos,

Isabel Sancho Carbonell y Daniel Sanchiz Castaño (eds.), 2016

(C) d'aquesta edició: Universitat d'Alacant

ISBN: 978-84-16724-00-0

Dipòsit legal: A 92-2016

Disseny de coberta: candela ink

Composició: Página Maestra (Miguel Ángel Sánchez Hernández)

Impressió i enquadernació: Guada Impresores

\section{unte \\ Unión de Editoriales
Universitarias Españolas \\ WWW.une.es
WWA}

Esta editorial es miembro de la UNE, cosa que garantiza la difusión y comercialización nacional y internacional de sus publicaciones.

Reservados todos los derechos. Cualquier forma de reproducción, distribución, comunicación pública o transformación de esta obra sólo puede ser realizada con la autorización de sus titulares, salvo excepción prevista por la ley. Diríjase a CEDRO (Centro Español de Derechos Repográficos, www.cedro.org) si necesita fotocopias o escanear algún fragmento de esta obra. 


\title{
LOS PROGRAMAS DE RETORNO VOLUNTARIO. EL PLAN TIERRAS DEL GOBIERNO DE ECUADOR Y SU INCIDENCIA EN ESPAÑA ${ }^{1}$
}

\author{
Juan Antonio Márquez Domínguez. \\ Instituto de Desarrollo Local. Departamento de Geografía, Universidad de Huelva \\ antonio@uhu.es \\ Mercedes Gordo Márquez \\ Instituto de Desarrollo Local. Departamento de Geografía, Universidad de Huelva \\ Rafael Viruela Martínez \\ Instituto de Desarrollo Local. Departamento de Geografía, Universidad de Huelva \\ Jesús Felicidades García \\ Instituto de Desarrollo Local. Departamento de Geografía, Universidad de Huelva
}

\section{RESUMEN}

España ha sido uno de los principales destinos elegidos en los proyectos migratorios internacionales de los ciudadanos que han partido de Ecuador. Las similitudes culturales y las perspectivas de empleo han permitido contrarrestar la distancia geográfica que separa ambos países. El éxito de unos atrajo a otros, entrando en funcionamiento los postulados de la teoría de redes. De esta forma, especialmente a partir de la década de 1990, los ecuatorianos se han convertido en uno de los colectivos de mayor peso en España, ocupando puestos en la construcción, las actividades agrícolas y el sector servicios, entre ellos en el servicio doméstico.

1 Este documento recoge parte del trabajo realizado en la ejecución del Proyecto I+D+i (20102014): "Gestión colectiva de contrataciones agrícolas en origen y sus soportes territoriales en España y Marruecos: propuesta de concatenación de campañas e implicaciones en el codesarrollo". Dicho Proyecto es financiado por el Ministerio de Ciencia e Innovación (Ref. CSO2010-18764, 2011-2014). Juan Antonio Márquez actúa como investigador principal y jefe de fila de las universidades que participan en el proyecto: Alicante, Valencia, Lérida, Jaén y Huelva. 
Con el paso del tiempo se han ido consolidando en el país. Los procesos extraordinarios de regularización aprobados por los distintos Gobiernos españoles han contribuido a promocionar su seguridad jurídica en España, aunque en algunos casos solo ha sido de manera temporal. También han aprovechado y contribuido al dinamismo mostrado por la economía española, que se traducía en una importante creación de empleo. En base a esta estabilidad han adquirido viviendas y se han acogido en muchos casos a la reagrupación familiar, trayendo a sus parejas, descendientes y ascendientes. Estos procesos dotaron al colectivo de una mayor vocación de permanencia en España, reforzado aún más si cabe por el hecho de que en muchos casos se han nacionalizado español. No obstante lo suelen hacer sin renunciar a su nacionalidad de origen y con el objeto de aprovechar los beneficios, la seguridad jurídica, que les reporta el ser considerado ciudadano de la Unión Europea.

La situación descrita de consolidación y crecimiento de la colonia ecuatoriana ha encontrado un punto de inflexión en los últimos años. La crisis económica que atraviesa España ha provocado que parte de la población ecuatoriana haya perdido su empleo. Los propios españoles vuelven a tomar el camino de la emigración ante el deterioro de la situación sociolaboral y la falta de perspectivas profesionales. En esta línea el Gobierno de España ha puesto en marcha diversos programas de retorno voluntario dirigidos a los extranjeros que se encuentran en el país, al objeto de relajar la presión sobre el mercado de trabajo y reducir la competencia laboral.

En un primer momento estos programas de retorno han tenido poco eco entre sus destinatarios. Sin embargo, la prolongación en el tiempo de la recesión, e incluso la acentuación la misma, ha llevado a que muchos hayan agotado las prestaciones por desempleo a que tenían derecho por el tiempo trabajado. Como resultado carecen de recursos económicos para hacer frente a las hipotecas que suscribieron para adquirir sus viviendas, son desahuciados y las perspectivas laborales no son nada halagüeñas a corto plazo. Ante esta situación el regreso al país de origen comienza a ganar adeptos. A ello hay que sumar que al contrario de lo que sucede en España, la economía de Ecuador se muestra dinámica, crece, y el Gobierno del país adopta también medidas para hacer atractivo el regreso de sus nacionales. Entre las citadas medidas se encuentra el Plan Tierra, dirigido a realizar una reforma agraria y dotar de tierras a la población para su puesta en valor. La presente comunicación se centrará en analizar la incidencia de este Plan entre los ecuatorianos que se encuentran en España.

Este documento recoge parte del trabajo realizado por los autores en la ejecución del proyecto I+D+i Gestión colectiva de contrataciones agrícolas en origen y sus soportes territoriales en España y Marruecos: propuesta de concatenación de campañas e implicaciones en el codesarrollo. Dicho proyecto es financiado por el Ministerio de Ciencia e Innovación (Ref. CSO2010- 
18764, 2011-2013) y está siendo llevado a cabo por investigadores de distintas universidades españolas. El "Instituto de Desarrollo Local" (IDL), grupo de investigación de la Universidad de Huelva, actúa como jefe de filas del mismo.

Palabras claves: retorno voluntario, los flujos migratorios, Plan Tierras del Gobierno de Ecuador.

\section{THE VOLUNTARY RETURN PROGRAMS. THE GOVERNMENT OF ECUADOR LAND PLAN AND ITS IMPACT ON SPAIN}

\section{Abstract}

Spain has been one of the top tourist destinations in international migration projects of citizens who have left Ecuador. Cultural similarities and employment prospects have offset the geographical distance separating the two countries. The success of some attracted others to come on stream the tenets of network theories. In this way, especially since the 1990s, Ecuatorians have become one of the groups with more weight in Spain, holding positions in construction, farming and the service sector, including domestic servants. With the pass of time they have been consolidated in the country. The extraordinary regularization process approved by the various Spanish governments have helped to promote legal security in Spain, although in some cases has only been temporary. They have also benefited and contributed to the dynamism of the Spanish economy, which resulted in significant job creation. Based on this stability they have acquired properties and have been welcomed in many cases of family reunion, bringing their partners, descendants and ascendants.

These processes endowed the collective greater degree of permanence in Spain, enhanced even further by the fact that in many cases have been nationalized as Spanish. Notwithstanding usually they do not renounce their nationality of origin in order to reap the benefits, legal security, reporting them to be considered a citizen of the European Union.

The described situation of consolidation and growth of the Ecuadorian colony has found a turning point in recent years. The economic crisis in Spain has caused part of the Ecuadorian population to lose their jobs.

The Spaniards themselves retake the path of emigration to the deterioration of social and working conditions and lack of career prospects. In this vein the Government of Spain has implemented various voluntary return programs targeting foreigners in the country, in order to ease the pressure on the labor market and reduce labor competition underway.

At first these return programs have had little echo among the recipients. However, the extension in time of recession, and even stress it, it has led many to have exhausted unemployment benefits they were entitled for the time worked. As a result, they lack the financial resources to meet the mortgages 
signed to acquire their homes, they are hopeless and job prospects are not bright in the short term. In this situation they return to the home country begins to gain followers. To this must be added that, contrary to what happens in Spain, Ecuador's economy is dynamic, is growing, and the government of the country also takes steps to appeal the return of their nationals. Among those measures is the Land Plan, aimed at making land reform and give land to the population for its value. This communication will focus on analyzing the impact of this plan among Ecuadorians who are in Spain.

This document contains part of the work done by the authors in the project implementation R \& D + i collective management of agricultural contracts in origin and territorial supports Spain and Morocco: concatenation proposed campaigns and co-development implications. This project is funded by the Ministry of Science and Innovation (Ref CSO2010-18764, 2011-2013) and is being conducted by researchers from different Spanish universities. The "Institute for Local Development" (IDL), a research group at the University of Huelva, acts as team leader.

Key words: voluntary return, flows migration, Plan Tierras del Gobierno de Ecuador.

\section{INTRODUCCIÓN}

La crisis económica y financiera global, iniciada a finales de 2007, afecta con especial virulencia a España, donde el paro se ha incrementado a gran velocidad. El desempleo afecta sobre todo a la mano de obra extranjera, cuya tasa supera el $39 \%$ frente al $25 \%$ de la población activa autóctona. El retorno es una de las múltiples estrategias que adoptan los migrantes para evitar los efectos de la recesión. Según la Estadística de Variaciones Residenciales, entre 2007 y 2011 cerca de 1,2 millones de extranjeros, con predominio de latinoamericanos (el 39\% del total), han abandonado España y la mayoría han retornado a sus países de origen.

Como ha ocurrido en otros contextos espaciales y temporales, España anima a los emigrantes a regresar a casa, con este motivo ha puesto en marcha programas de retorno: el voluntario, en vigor desde 2008, y el productivo, que se puso en marcha en 2010. El Plan de Tierras, impulsado a principios de 2013 por el gobierno de Ecuador, se inserta dentro de este último.

La incidencia que tienen las políticas en el retorno es anecdótica ya que, por ejemplo, entre 2009 y 2011 menos de 5.000 ecuatorianos se acogieron a estos programas. Los emigrantes que lo hicieron en 2011 apenas supusieron el $3 \%$ de los flujos de retorno. 


\section{RETORNO DE INMIGRANTES}

El retorno de inmigrantes a sus países de origen ha sido observado como una meta social y económica de numerosas políticas demográficas, que han visto en ello, además de una acción humanitaria, una posible reactivación de territorios, especialmente los de usos vacantes. El retorno de los inmigrantes a sus países de origen se ha planteado en momentos de crisis en los países de acogida y se ha pretendido que sea una vuelta productiva, ligando el retorno a determinadas actividades, generalmente agrarias.

Con frecuencia, los intentos de reforma agraria en espacios vacantes o de frontera han sido los soportes de las políticas de retorno productivo y en torno a ellas se han montado "metanarraciones" sobre los objetivos de Estados, que se preocupan por los ciudadanos que viven dentro y fuera del país. Esto ha argumentado políticas de retorno, tanto por parte de los países de acogida como de origen que han colaborado de distintas formas para planificar una vuelta ordenada. $^{2}$

Sin embargo, por desgracia, muchas de estas intervenciones de retorno productivo han quedado en el camino o han cosechado un estrepitoso fracaso, como lo fue el Plan Almonte-Marisma en las inmediaciones del Parque Nacional de Doñana, donde trabajadores andaluces que residían en Holanda regresaron en 1979 a España para dedicarse a la explotación colectiva de una finca agropecuaria, la cooperativa Pequeña Holanda, comprada con ayuda de los Gobiernos de ambos países. "Para el Gobierno holandés se trataba de ensayar, a un precio módico, formas nuevas y sugestivas de quitarse de en medio el excedente de población activa extranjera sobrante por culpa de la crisis; por eso ofreció sesenta millones de pesetas de subvención y no tuvo inconveniente en llegar hasta los ochenta cuando se le demostró su necesidad. Puestos en contacto los emigrantes con las autoridades españolas, el Instituto Nacional de Reforma y Desarrollo Agrario -Iryda- elaboró un informe técnico y financiero sobre las posibilidades que para el proyecto en cuestión ofrecía la finca llamada Najarsa, en el término municipal de Almonte en la provincia de Huelva" (El País, 1979). Hoy, después de múltiples vicisitudes y abandono de la explotación primitiva, la Pequeña Holanda subsiste en Arcos de la Frontera, provincia de Cádiz, donde se dedica a la producción y comercialización de productos agrícolas, que nada tiene que ver con el proyecto inicial.

2 La Fundación INCYDE, Instituto Cameral para la Creación y Desarrollo de la Empresa, es el organismo beneficiario de la subvención de Retorno Productivo, concedida por el Ministerio de Empleo y Seguridad Social, con cargo a los Presupuestos Generales del Estado. Para tal fin, INCYDE ha centrado sus actuaciones en la formación empresarial de aquellos inmigrantes que, encontrándose en situación legal en España, quieran retornar a sus países de origen con un plan de empresa. La Fundación INCYDE se dirigirá al colectivo procedente de Argentina, Brasil, Colombia, Ecuador, Paraguay y Perú, centrando sus actividades tanto en hombres como en mujeres, sin realizar ningún tipo de discriminación. 


\section{EXTRANJEROS EN ESPAÑA}

En la primera década del siglo XXI, España registró un notable aumento de la inmigración. Más de cinco millones de personas venidas del extranjero han enriquecido y movilizado el mercado de trabajo y la demografía española. El colectivo procedente de Ecuador es uno de los más importantes, ocupando en 2004 el primer puesto entre la población foránea (figura 1). Sin embargo, en los últimos años, las cifras disminuyen, al principio de forma lenta y de forma acelerada en fecha reciente, de manera que en la actualidad hay poco más de 262.000 residentes de nacionalidad ecuatoriana.

Pero la llegada de inmigrantes, más que el mero hecho económico o espacial, tienen un largo alcance porque la llegada de extranjeros trajo fuerza laboral a los yacimientos de empleo no cubiertos por nacionales. Ello supuso:

1. Un relevo a una población agraria española envejecida, que no ha encontrado repuesto generacional español en las actividades agrarias.

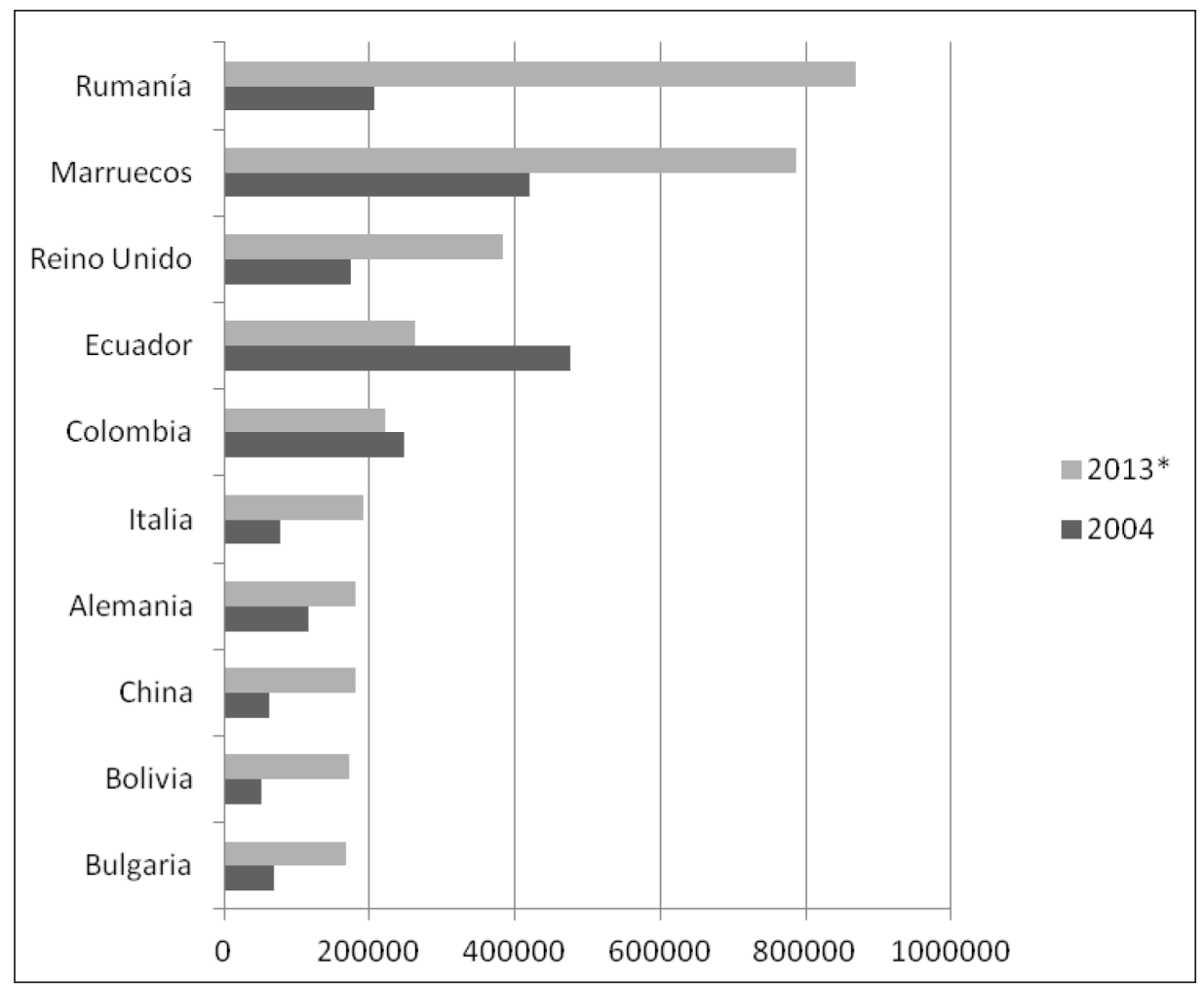

Figura 1. Colectivos extranjeros con mayor número de residentes en España

Fuente: INE: Padrón de Habitantes. Datos a 1 de enero de 2013 y 1 de enero de 2004. 
2. Una relocalización del origen geográfico del trabajo agrario, predominantemente española hasta los años 90 y extranjera a partir del 2000.

3. Cambios en las estrategias de aprovisionamiento de mano de obra, en la búsqueda de diferencias salariales mayores entre los lugares de origen y de trabajo.

Cuadro 1. Cupos para trabajadores extranjeros aprobados en los Acuerdos de contingente

\begin{tabular}{|c|c|c|c|}
\hline CONTINGENTE & ESTABLES & TEMPORADA & TOTAL \\
\hline 1993 & 10.100 & 10.500 & 20.600 \\
\hline $1994(1)$ & S. E. & S. E. & 37.600 \\
\hline 1995 & S. E. & S. E. & 8.000 \\
\hline 1996 & \multicolumn{3}{|c|}{ No se abrió el contingente } \\
\hline $1997(2)$ & S. E. & S. E. & 24.690 \\
\hline 1998 & S. E. & S. E. & 28.000 \\
\hline 1999 & S. E. & S. E. & 30.000 \\
\hline 2000 & \multicolumn{3}{|c|}{ No se abrió el contingente } \\
\hline 2001 & \multicolumn{3}{|c|}{ No se abrió el contingente } \\
\hline 2002 & 10.884 & 21.195 & 32.079 \\
\hline 2003 & 10.575 & 23.582 & 34.157 \\
\hline 2004 & 10.908 & $20.070(3)$ & $30.978(3)$ \\
\hline $2005(4)$ & 6.594 & 0 & 6.594 \\
\hline 2006 & 16.878 & S. E. & S. E. \\
\hline 2007 & 27.034 & $61.000(5)$ & S. E. \\
\hline 2008 & 15.731 & S. E. & S. E. \\
\hline 2009 & 901 & S. E. & S. E. \\
\hline
\end{tabular}

S. E.: Sin especificar.

(1) En un principio se aprobó un cupo de 20.600 autorizaciones. Sin embargo, el Acuerdo de contingente para el año 1995 aprobó una extensión del contingente de 1994 cifrada en un máximo de 17.000 autorizaciones, recogiendo además del propio cupo el año 1995.

(2) Inicialmente se aprobaron 15.000 autorizaciones, pero después se amplió hasta 24.690.

(3) En el caso de las ofertas de temporada, el Acuerdo de contingente para este año sólo publica las de carácter genérico.

(4) No supone un contingente nuevo, sino una prórroga del establecido para el año 2004 respecto a los puestos que habían quedado vacantes.

(5) Es una previsión de las contrataciones de duración determinada, no sólo del contingente de temporada.

Fuente: Acuerdo de contingente para cada uno de los años. Elaboración: Gordo, M., 2009. 
4. Alto grado de dependencia de la fuerza de trabajo extranjera, muchas veces imprescindible para la existencia de las agriculturas de frontera y los sectores de servicios ligados al turismo.

5. Fuerte incidencia a corto y medio plazo en las estructuras demográficas locales y enorme influencia, a largo plazo, en la geografía y demografía de la población española.

Aunque existe notable presencia de extranjeros en España por motivos de ocio, los mayores colectivos de inmigrantes tienen motivos laborales: Rumanos, marroquíes, ecuatorianos y colombianos.

Muchos de estos inmigrantes laborales trabajan en el campo, a los que habría que añadir los que vienen con contratos en origen de forma coyuntural. Antes de la crisis, la mayor parte de lpps inmigrantes se concentraban en áreas litorales y Madrid-Barcelona. En las zonas costeras fueron imprescindibles en las explotaciones agrarias y en mantenimiento de la exportación hortofrutícola. En 2007, España ofertó más de 70.000 cupos de trabajo temporal para jornaleros extranjeros y mantuvo a más de 150.000 Trabajadores extranjeros afiliados en alta laboral al sistema de la Seguridad Social por el sistema agrario.

El importante volumen de exportación de alimentos y de importación de fuerza laboral, aún mantenida con la crisis de 2008 ..., descubre una fortaleza agrícola en diversos sistemas productivos locales que ofrecen un precioso yacimiento de empleo a jornaleros nacionales y extranjeros y amplias oportunidades de agronegocios.

Sin embargo, la crisis de 2008, ya suficientemente conocida por su impacto en el paro, generó un enrarecimiento de los yacimientos de empleo porque la población nacional consideró, especialmente en la agricultura, que la población extranjera ocupaba "puestos de trabajo", para los que los nacionales deberían tener preferencia. En los últimos años, el sector agrario de diversas comarcas y regiones acoge a trabajadores españoles procedentes de otros sectores de actividad (Torres y Gadea, 2010; Menor, 2011; Bernat y Viruela, 2011). Esta "actitud social y política", se vio muy pronto reflejada en la contratación en origen, que pasó por ejemplo, en la provincia de Huelva, termómetro de la contratación en origen en España, de los más de 40.000 efectivos a los 2.185 de la campaña 2012-2013.

\section{ECUATORIANOS EN EsPaÑA}

Los 390.365 ecuatorianos con tarjeta de residencia en vigor a finales de 2012 constituyen el tercer colectivo por importancia tras rumanos y marroquís. 


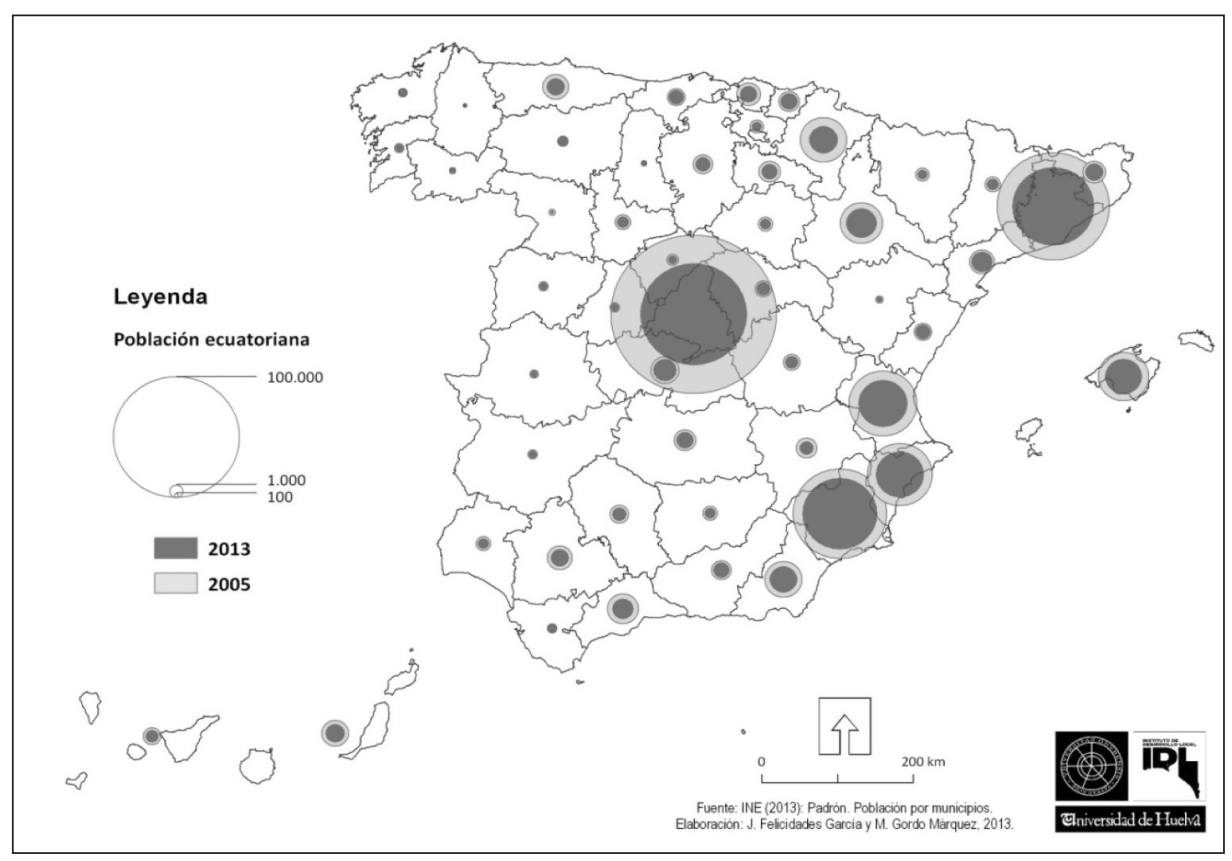

Mapa 1. Ecuatorianos en España según datos del Padrón Municipal (datos a 1 de enero).

Fuente: elaboración propia.

Los ecuatorianos se localizan principalmente en las provincias de Madrid, Barcelona y Murcia, aunque mantiene importantes colectivos en casi todas las provincias españolas.

La inmigración ecuatoriana, a pesar de sus dimensiones, es una de las mejor integrada porque, la cercanía cultural del ecuatoriano incide en el sobredimensionamiento de la concesión de nacionalidad española, de tal suerte que las citadas concesiones de nacionalidad puede poner en peligro el retorno productivo, ya que muchas veces las probabilidades de inserción en Ecuador, no logran vencer la inercia de ecuatorianos que han echado sus raíces en España. Además, la legislación española permite a los ecuatorianos obtener la nacionalidad "de forma expedita", con la justificación de dos años de residencia en España.

Así, en el rastreo de concesiones de nacionalidad realizado desde 2007 hasta 2011, se observa como los ecuatorianos son los mayores beneficiados de la nacionalidad española y con una tendencia al aumento. De esta forma, si en 2007 fueron 21.371, en 2011 superaron la cifra de 32.000. A mucha distancia les siguen colombianos y marroquís, con 19.803 y 14.427 nacionalizaciones en 2011. 
5. CONCESIÓN DE NACIONALIDAD ESPAÑOLA 2007-2011

Cuadro 2. Concesión de nacionalidad española entre los años 2007-2011

\begin{tabular}{|l|r|l|r|l|r|}
\hline PAÍS & $\mathbf{2 0 0 7}$ & PAÍS & $\mathbf{2 0 0 9}$ & PAÍS & $\mathbf{2 0 1 1}$ \\
\hline Ecuador & 21.371 & Ecuador & 25.769 & Ecuador & 32.026 \\
\hline Colombia & 13.852 & Colombia & 16.527 & Colombia & 19.803 \\
\hline Marruecos & 7.864 & Marruecos & 6.683 & Marruecos & 14.427 \\
\hline Perú & 6.490 & Perú & 6.368 & Perú & 9.255 \\
\hline Argentina & 4.810 & Argentina & 4.629 & Argentina & 5.482 \\
\hline Rep. Dominicana & 2.800 & Rep. Dominicana & 2.766 & Bolivia & 5.333 \\
\hline Cuba & 2.466 & Cuba & 2.696 & Rep. Dominicana & 4.985 \\
\hline Venezuela & 1.324 & Bolivia & 1.813 & Cuba & 3.088 \\
\hline Filipinas & 872 & Venezuela & 1.744 & Venezuela & 2.596 \\
\hline Uruguay & 839 & Uruguay & 1.451 & Uruguay & 1.978 \\
\hline Chile & 838 & Chile & 1.090 & Brasil & 1.854 \\
\hline Brasil & 779 & Brasil & 943 & Chile & 1.556 \\
\hline Bolivia & 709 & México & 584 & Portugal & 884 \\
\hline México & 593 & Portugal & 485 & Paraguay & 864 \\
\hline Gambia & 442 & Filipinas & 431 & México & 856 \\
\hline Portugal & 381 & Gambia & 396 & Nigeria & 670 \\
\hline Senegal & 378 & China & 337 & Filipinas & 612 \\
\hline $\begin{array}{l}\text { Guinea Ecuato- } \\
\text { rial }\end{array}$ & 354 & Paraguay & 298 & Senegal & 577 \\
\hline China & 310 & Senegal & 289 & Argelia & 544 \\
\hline Argelia & & & Puinea Ecuato- & 491 \\
\hline
\end{tabular}

Fuente: http://extranjeros.empleo.gob.es/es/Estadisticas/operaciones/concertificado/201212/ Residentes_Principales_Resultados_31122012.pdf

En España residen cerca de 400.000 ciudadanos ecuatorianos para los que el Gobierno está implementando medidas de apoyo y planes de regreso al país de forma productiva y ordenada.

El retorno productivo es una estrategia para inmigrantes que trata de orientar la vuelta a sus países de origen, dotando a los inmigrantes de instrumentos y capacidades que le permitan una inserción sin traumas. En España la Fundación Incyde, Instituto Cameral para la Creación y Desarrollo de la Empresa, desde 
el Ministerio de Empleo y Seguridad Social lidera el Programa de Retorno Productivo para inmigrantes. INCYDE se dirigirá al colectivo procedente de Argentina, Brasil, Colombia, Ecuador, Paraguay y Perú, centrando sus actividades en facilitar, poner en marcha y desarrollar un negocio en el país de origen.

El inmigrante interesado en retornar a su país de procedencia con un proyecto productivo, podrá participar en los programas de formación de la Fundación INCYDE, por medio del cual recibirá tutorización individualizada y asistirá a clases conjuntas, de las áreas fundamentales para la elaboración de un plan de empresa. De esta manera el alumno irá avanzando en las distintas etapas del proyecto hasta que pueda elaborar un documento completo sobre su plan de empresa.

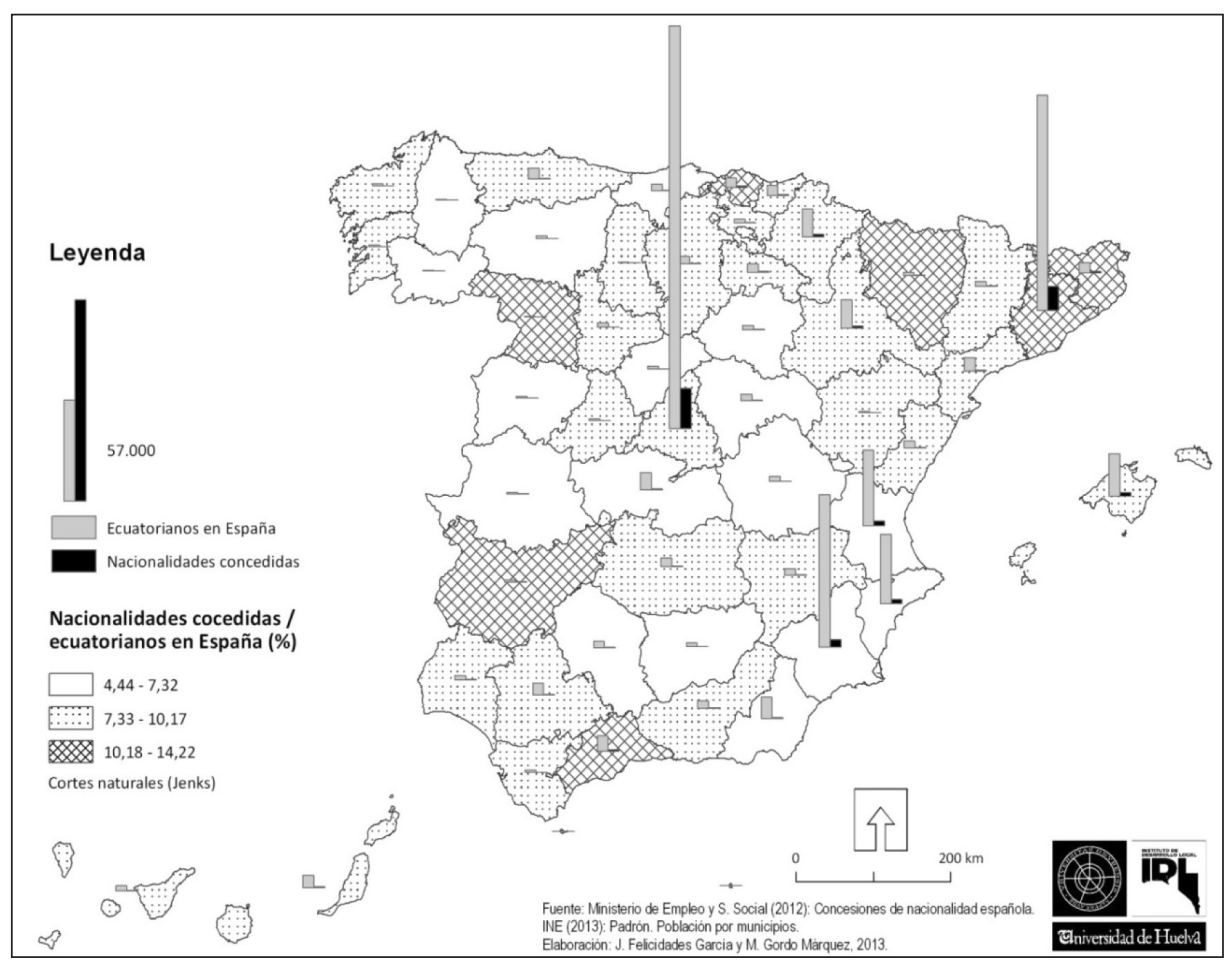

Mapa 2. Concesiones de nacionalidad española a ecuatorianos por provincia del Registro Civil, 2011.

Fuente: elaboración propia.

Las áreas en que se impartirán formación serán las siguientes: a. Análisis del entorno, b. Área Financiera, c. Área Comercial, d. Entorno Jurídico y Fiscal, e. Nuevas Tecnologías, Internet y Comercio Electrónico, f. Entorno laboral y g. Comunicación" (Incyde, 2013). 
Para ello, entre otros instrumentos, Incyde posee un "Vivero flotante" para trabajar en la puesta en marcha de la actividad empresarial. El futuro retorno se irá fortaleciendo y madurando con un plan de empresa apoyada por expertos consultores, y contactos con empresas, que le permitirán establecer sinergias y enfrentar el futuro.

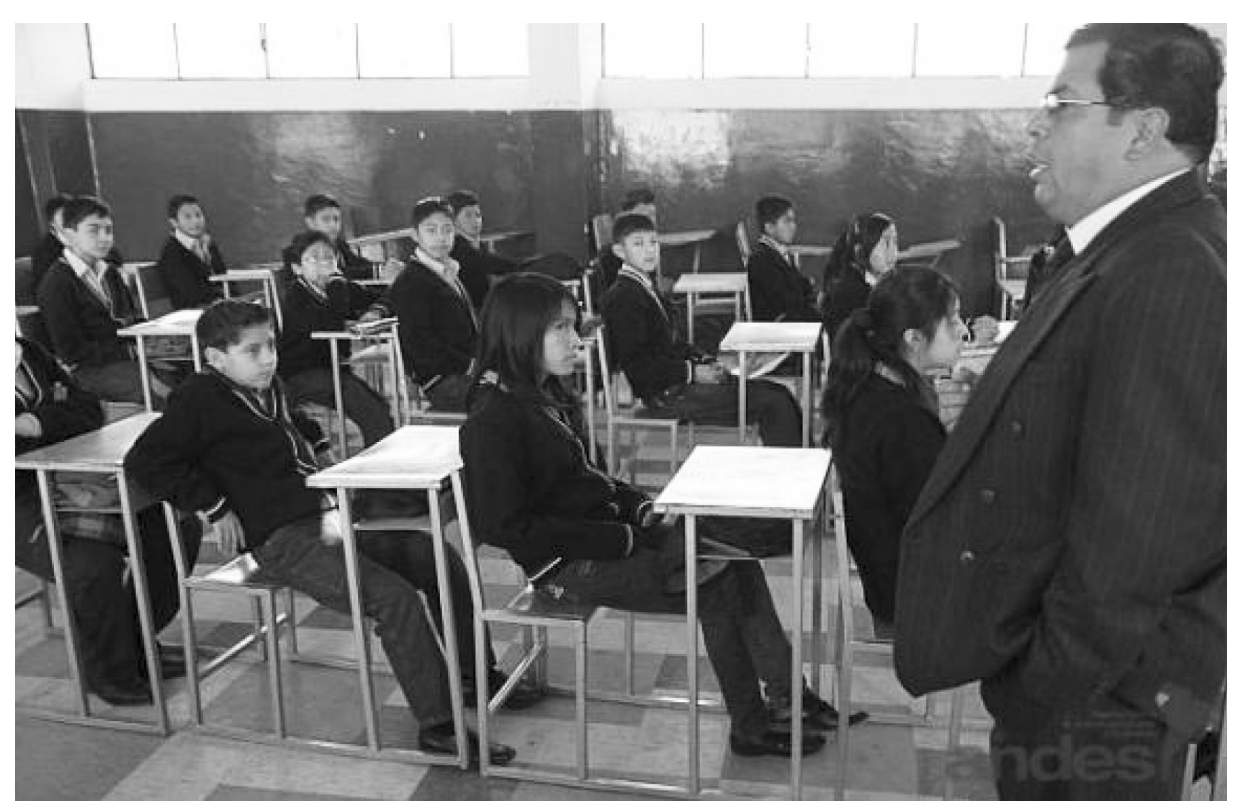

Los maestros ecuatorianos.

Fuente: www.andes.info (2013): Los maestros ecuatorianos que viven en España podrán aplicar a los concursos de méritos y oposición para ocupar las plazas vacantes del magisterio fiscal en Ecuador, en http://www.andes.info.ec/es/sociedad/ecuador-enfoca-retorno-productivo. En línea 15.06-2013.

\section{Plan Tierras}

El Plan Tierras de Ecuador tiene como objetivo construir un modelo de desarrollo rural basado en la economía social solidaria, que garantice la soberanía y seguridad alimentarias y el buen vivir en el marco de la participación social. (Gobierno Ecuador: 2013).

Es un macroemprendimiento del gobierno ecuatoriano, encargado de la redistribución de las tierras, en el contexto de una auténtica Revolución Agraria. Ya en marzo del 2013 tenía adjudicadas 20.524 hectáreas que han beneficiado a más de 4.000 familias.

El proyecto se extiende por todo el Ecuador, pero actúa con más intensidad en las provincias del Guayas, Los Ríos, El Oro, Azuay, Cañar, Chimborazo, 
Cotopaxi y Tungurahua, territorios donde existen mayores dificultades de acceso a la tierra.

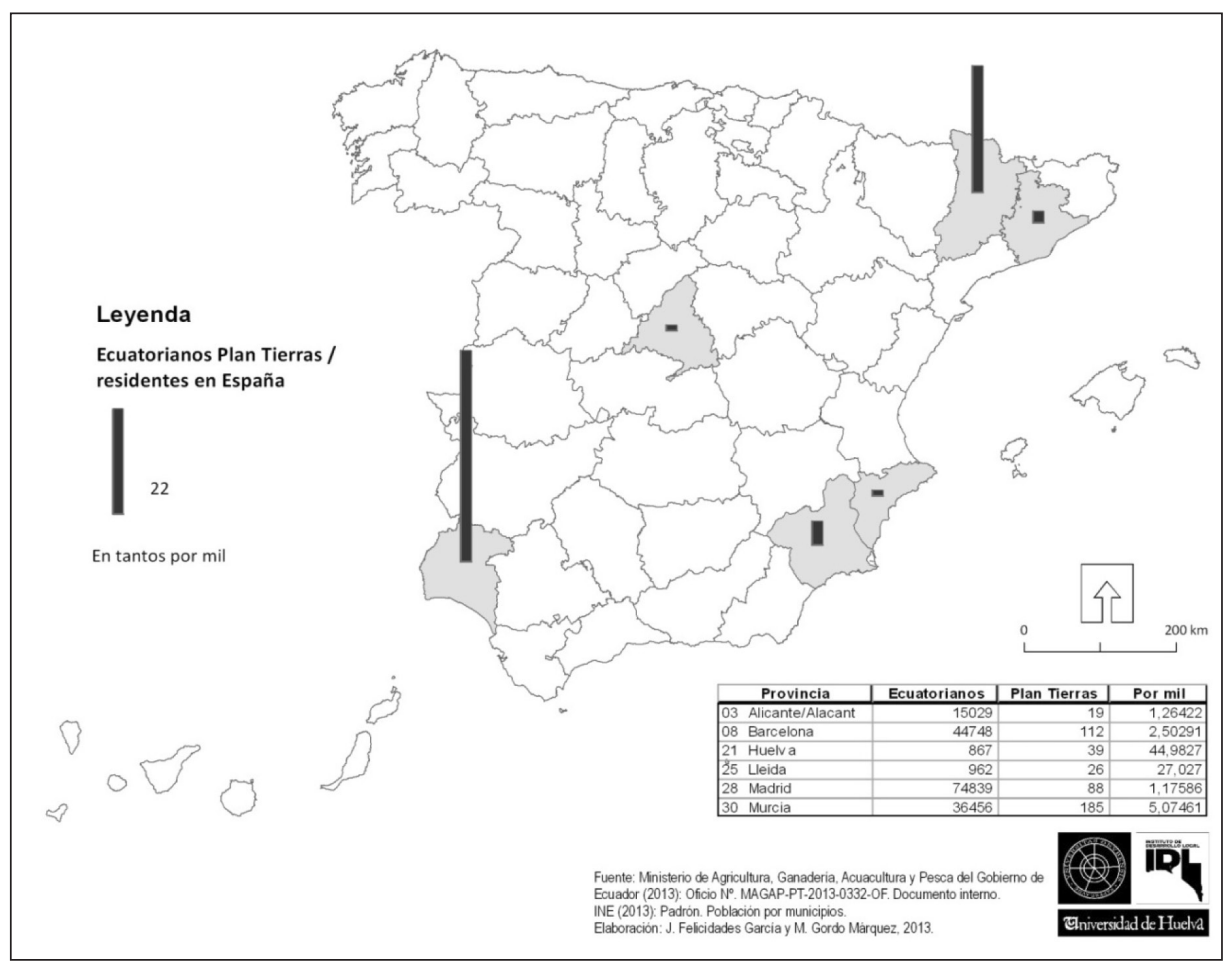

Mapa 3. Ecuatorianos en España acogidos al Plan Tierras (datos a 25 de marzo de 2013).

Fuente: elaboración propia.

El Plan de Tierras tiene como metas:

- Consolidar los procesos de redistribución de la tierra

- Fomentar la participación social en los procesos de redistribución de la tierra

- Fortalecer organizativamente a los actores involucrados en el Plan Tierras.

- Conformar redes productivas que garanticen la reproducción de la producción a través de la comercialización, intercambio y consumo, en el marco de la economía social y solidaria.

- Velar porque no se produzcan prácticas de concentración o fragmentación de las tierras adjudicadas. 
Estas metas se tratan de cumplir desde 7 ejes estratégicos:

Para cumplir las metas se están ejecutando siete ejes estratégicos del Plan Tierras

1. Distribución de predios estatales

2. Titulación de territorios indígenas

3. Creación de un fondo de tierras eje

4. Expropiación de tierras en áreas priorizadas

5. Sistema de catastro de tierras rurales

6. Consolidación parcelaria

7. Nueva legislación agraria y mecanismos de control de fragmentación y reconcentración

Como complemento del Retorno Productivo, el Gobierno del Ecuador, está impulsando en España el retorno de pequeños y medianos agricultores.

Efectivamente, Gobierno del Ecuador a través de la Embajada del Ecuador en España ha puesto en marcha el denominado Plan Tierras. El proyecto está dirigido a la población ecuatoriana residente en España y se enmarca dentro de las políticas gubernamentales de planes de retorno.

Al igual que ya se hizo con el colectivo de médicos especialistas residentes en España, el Gobierno del Ecuador se plantea ahora ofrecer la posibilidad de generar emprendimiento entre los agricultores ecuatorianos residentes en España.

El objetivo de esta iniciativa, es ofrecer parcelas de cultivo a colectivos organizados interesados en aplicar los conocimientos adquiridos en España en el sector agrario.

El proyecto se presentó en Enero en las ciudades de Lleida y Murcia; Madrid, Barcelona, Huelva y Almería.

El taller que se llevó a cabo en Huelva puede ser considerado como prototipo: tuvo una elevada participación y se inscribieron unas 120 familias asentadas en toda la provincia de Huelva. En cada taller, una delegación de técnicos y funcionarios del Ministerio de Agricultura del Ecuador se reunirán con agricultores y profesionales del sector para conformar las asociaciones agrícolas, explicar las líneas de financiamiento y crédito, y coordinar los proyectos de emprendimiento. En esta primera fase del Plan Tierras, el Gobierno del Ecuador prevé beneficiar a unas 300 personas aproximadamente. (Embajada: 2013).

La propuesta se fraguó el año 2012, tras celebrarse varios encuentros con la comunidad ecuatoriana que trabaja en el sector agropecuario, proponiéndose la extensión del Plan Tierras a los ciudadanos residentes en España, dado que la crisis iniciada en 2008 en España ha dejado a muchos ecuatorianos en el paro. 


\section{BIBLIOGRAFÍA}

BERNAT, J. S. y VIRUELA, R. (2011): "The Economic Crisis and Immigration: Romanian Citizens in the Ceramic Tile District of Castelló (Spain)", Journal of Urban and Regional Analysis, vol. 3, n ${ }^{\circ}$ 1, pp. 45-65, en http:// www.jurareview.ro/2011_1_1/a_2011_1_1_4_bernat.pdf, en línea 22 de marzo de 2013.

EL PAÍS (1979): "La Pequeña Holanda, polémica cooperativa agrícola al borde de Doñana", en http://elpais.com/diario/1979/11/17/. En línea 10-05-2013.

EMBAJADA ECUADOR (2013): El gobierno del Ecuador impulsa en España el plan tierras para facilitar el retorno productivo de sus ciudadanos. Embajada del Ecuador En España. Boletín de Prensa No 1.404. Fecha: 15/01/2013.

GOBIERNO ECUADOR: (2013): Plan Tierra, en http://www.agricultura.gob. ec/plan-tierras/. En línea 15-05-2013.

GORDO, M. y MÁRQUEZ, J.A. (2009): Temporeros extranjeros en Huelva (España) con contratos en origen. Colombianos y ecuatorianos, en Orbis Incognitus. Avisos y Legajos del Nuevo Mundo. Universidad de Huelva, pp. 765-780.

GOBIERNO DE ESPAÑA (2013): Extranjeros y empleo, en http://extranjeros. empleo.gob.es... En línea 15-05-2013.

INCYDE (2013): Programa de Retorno Voluntario Productivo. Fundación Incyde Instituto Cameral para la Creación y Desarrollo de la Empresa. Madrid.

INE (2013): Padrón. Población por municipios. www.ine.es. En línea 15-052013.

MAGAP (2013): Ecuatorianos en España acogidos al Plan Tierra. Ministerio de Agricultura, Ganadería, Acuicultura y Pesca del Gobierno del Ecuador. Documentación interna.

MÁRQUEZ, J.A. (2009): "Migraciones circulares. Contratos en origen y codesarrollo", en Inmigración y Desarrollo Regional. Universidad de Almería, pp. 253-264.

MENOR, J. (2011): La movilidad de los inmigrantes temporeros para las campañas agrícolas: el caso de los marroquíes durante la recogida de la aceituna en la provincia de Jaén. In García, F. J. y Kressova, N. (coord.): Actas del I Congreso Internacional sobre Migraciones en Andalucía. Granada, Instituto de Migraciones, pp. 543-558.

MINISTERIO DE EMPLEO Y SEGURIDAD SOCIAL (2013): Concesiones de nacionalidad española. Madrid.

SUBDELEGACIÓN DEL GOBIERNO EN HUELVA (2013): Contratos en origen. Documentación interna. 
WWW.ANDES.INFO (2013): Los maestros ecuatorianos que viven en España podrán aplicar a los concursos de méritos y oposición para ocupar las plazas vacantes del magisterio fiscal en el país, en http://www.andes.info. ec/es/sociedad/ecuador-enfoca-retorno-productivo. En línea 15.06-2013

TORRES, FRANCISCO y GADEA, Ma ELENA (2010): "Inserción laboral de los inmigrantes. Estructura etno-fragmentada y crisis económica. El caso del Campo de Cartagena (Murcia)", Sociología del Trabajo, nº 69, p. 73-94. 